\title{
Toward a comprehensive, theoretical model of compassion fatigue: An integrative literature review
}

\author{
Siedine K. Coetzee PhD, $\mathrm{RN}^{1}$ (1) | Heather K.S. Laschinger PhD, $\mathrm{RN}^{2 *}$
}

\author{
${ }^{1}$ School of Nursing Science, North-West \\ University, Potchefstroom, South Africa \\ ${ }^{2}$ Arthur Labatt Family School of Nursing, \\ University of Western Ontario, London, \\ Ontario, Canada

\section{Correspondence} \\ Siedine K. Coetzee, North-West University \\ (Potchefstroom Campus), School of Nursing \\ Science, 11 Hoffman Street, Potchefstroom \\ 2531, South Africa. \\ Email: siedine.knobloch@nwu.ac.za \\ Funding information \\ INDEN/STTI, Grant/Award number: 2012 \\ Post-doctoral Fellowship; National Research \\ Foundation of South Africa, Grant/Award \\ number: 84160
}

\begin{abstract}
This study was an integrative literature review in relation to compassion fatigue models, appraising these models, and developing a comprehensive theoretical model of compassion fatigue. A systematic search on PubMed, EbscoHost (Academic Search Premier, E-Journals, Medline, PsycINFO, Health Source Nursing/Academic Edition, CINAHL, MasterFILE Premier and Health Source Consumer Edition), gray literature, and manual searches of included reference lists was conducted in 2016 . The studies $(n=11)$ were analyzed, and the strengths and limitations of the compassion fatigue models identified. We further built on these models through the application of the conservation of resources theory and the social neuroscience of empathy. The compassion fatigue model shows that it is not empathy that puts nurses at risk of developing compassion fatigue, but rather a lack of resources, inadequate positive feedback, and the nurse's response to personal distress. By acting on these three aspects, the risk of developing compassion fatigue can be addressed, which could improve the retention of a compassionate and committed nurse workforce.
\end{abstract}

\section{KEYWORDS}

compassion fatigue models, compassion satisfaction, empathy, nursing practice environment

\section{1 | INTRODUCTION}

For a term that only emerged in the early 1990s, a tremendous amount of research has been done on compassion fatigue. Compassion fatigue has been studied across the globe (Ledoux, 2015), among most caregiving professions, including nurses and midwives, genetic workers, emergency service workers, chaplains, physicians, psychologists and social workers (Turgoose \& Maddox, 2017).

Compassion fatigue refers to the disengagement of caregivers from their patients, which culminates in a reduction or inability to feel empathy and compassion toward patients and an inability to provide the patient care that is deemed appropriate. It is the loss of meaningful and purposeful interaction between caregivers and patients (Coetzee \& Klopper, 2010; Ledoux, 2015). The term "compassion fatigue" was first publicly used by Joinson in 1992 when investigating the nature of burnout among nurses in an emergency unit. The concept was never formally defined, but described as having "lost the

*Deceased ability to nurture" (Joinson, 1992, p. 119). By 1995, the concept had been adopted by various caregiving professions, and was used as a replacement term for almost every occupational stress outcome related to caring professions, including vicarious traumatization, secondary trauma syndrome, secondary traumatic stress (STS), countertransference, SDS disorder, burnout and helper stress (Coetzee \& Klopper, 2010; Ledoux, 2015).

Because of the ambiguity of the term, research on compassion fatigue suffered from both conceptual and methodological limitations. This in turn hindered measurement, empirical research, and theoretical understanding of the term (Ledoux, 2015; Sabo, 2006). It was only in 2006, when Sabo first discussed compassion fatigue in relation to compassion and empathy that researchers began questioning the absence of the core constructs of compassion and fatigue in the definition of the concept. This provided the necessary impetus for further development of compassion fatigue research through concept analyses (Coetzee \& Klopper, 2010; Jenkins \& Warren, 2012; Lynch \& Lobo, 2012) that indicated the validity of the term "compassion fatigue" and its distinctness from other similar occupational stress outcomes. 
Some authors maintain that the lack of conceptual clarity has limited measurement, empirical study, and the development of a theoretical model of compassion fatigue (Ledoux, 2015). However, instruments to measure compassion fatigue (Figley, 1995; Stamm, 2010) and models to enhance theoretical understanding of compassion fatigue have been developed (Coetzee \& Klopper, 2010; Fernando \& Consedine, 2014; Figley, 1995, 2002; Geoffrion, Morselli, \& Guay, 2015; Jenkins \& Warren, 2012; Klimecki \& Singer, 2011; Lynch \& Lobo, 2012; Stamm, 2010; Valent, 1995, 2002).

These models have advanced the field of compassion fatigue research and share many similarities, often building on the work of pioneers in the field. Others add supplemental, unique, or conflicting aspects. The aspect causing the most conflict in the theoretical understanding of compassion fatigue is the role of empathy in the etiology of either compassion fatigue or compassion satisfaction. Pioneering theorists (Figley, 1995, 2002) have argued that empathy, although necessary to form the therapeutic relationship and provide care, is the main antecedent of compassion fatigue. This implies that if nurses are empathic and engage in the act of caring, then they will inevitably experience compassion fatigue, which is an erroneous idea to some scholars (Ledoux, 2015; Sabo, 2006, 2011). Empirical studies have also been equivocal, linking empathy to either the development of or protection from compassion fatigue (Turgoose \& Maddox, 2017), although several studies link empathy to improved patient outcomes (Derksen, Bensing, \& Lagro-Janssen, 2013) and nurse (Van Dam, Sheppard, Forsyth, \& Earleywine, 2011; Wagaman, Geiger, Shockley, \& Segal, 2015). However, the study of empathy by social neuroscientists during the past decade sheds light on the neural processes underlying empathy (Decety \& Lamm, 2009; GonzalezLiencres, Shamay-Tsoory, \& Brune, 2013), and provides the empirical evidence needed to critique current theoretical models of compassion fatigue and to develop a comprehensive theoretical model.

\section{1 | Study aim}

The aim of this study was perform an integrative literature review in relation to compassion fatigue models, appraise these models, and develop a comprehensive theoretical model of compassion fatigue.

\section{2 | METHODS}

For this study, an integrative literature review was selected as the method to effectively identify and appraise compassion fatigue models. A systematic and comprehensive search was conducted using the following keywords: "compassion fatigue" AND "model" OR "conceptual framework" OR "theory" to search electronic databases (PubMed, EbscoHost [Academic Search Premier, E-Journals, Medline, PsycINFO, Health Source Nursing/Academic Edition, CINAHL, MasterFILE Premier and Health Source Consumer Edition]). The electronic database searches were supplemented with manual searching of the reference lists of included studies. A search of gray literature, including Internet sites, was also carried out.

The inclusion criteria required the articles to be empirical or theoretical studies that described a model or conceptual framework or theory of compassion fatigue, and published between January 1992 (when the concept was first coined) and December 2016. Studies published in a language other than English were excluded.

The initial search yielded a total of 495 studies, the titles and abstracts of which were screened to determine those that were potentially eligible for inclusion. At this point, 244 studies were found to be duplicates, and 236 titles and abstracts were found to not be relevant, leaving 15 studies, the full texts of which were examined in detail.

Following this process, five studies were removed, as they did not meet the inclusion criteria. A manual search of the reference lists of the included studies and gray literature was conducted, and one study from each was added. The quality of each study was assessed using the John's Hopkins Nursing Evidence Based Practice: NonResearch Evidence Appraisal Tool for literature reviews and expert opinions. The checklist is comprised of five items for the literature review appraisal, and four items for the expert opinion appraisal. Each study was rated against the items of the checklist and achieved an overall score between 0 and 5 , which was then converted to a percentage score (0-100\%). Studies that denoted a percentage of $\geq 75 \%$ were included in the study. One study was excluded after critical appraisal, and a total of 11 studies were included in the review (Figure 1).

\section{3 | RESULTS}

This review identified 11 studies, which discussed seven models and three concept analyses of compassion fatigue. Of these seven models, five were published in the past decade. The models were developed by psychologists $(n=5)$, physicians $(n=1)$, and nurses $(n=1)$. All the concept analyses were developed by nurses and published in the past decade. The search produced much information about the current models of compassion fatigue and allowed critique of the models with regard to their strengths and limitations (Table 1).

\section{1 | Current models of compassion fatigue}

The compassion stress and fatigue model of Figley $(1995,2002)$ is centered on the assumption that empathy and emotional energy are required as a basis to connect with clients and respond to clients' pain. In this causal model, Figley explains that the entire process begins with exposure to the client, followed by empathic concern and empathic response based on the empathic ability of the caregiver. Empathic ability is the cornerstone of the model and refers to the caregiver's aptitude for noticing the pain of others. The greater the caregiver's empathic ability, the greater his/her empathic concern ("motivation to respond to people in need") (Figley, 2002, p. 1436) and empathic response ("an effort to reduce the suffering of the sufferer") (Figley, 2002, p. 1436), but equally so, the ability to empathize renders the caregiver vulnerable to compassion fatigue. This model then further cascades into residual compassion stress, which can be minimized by one of two coping actions: either a sense of satisfaction or disengagement. However, with prolonged exposure, traumatic memories that elicit an emotional response, and other degrees of life 


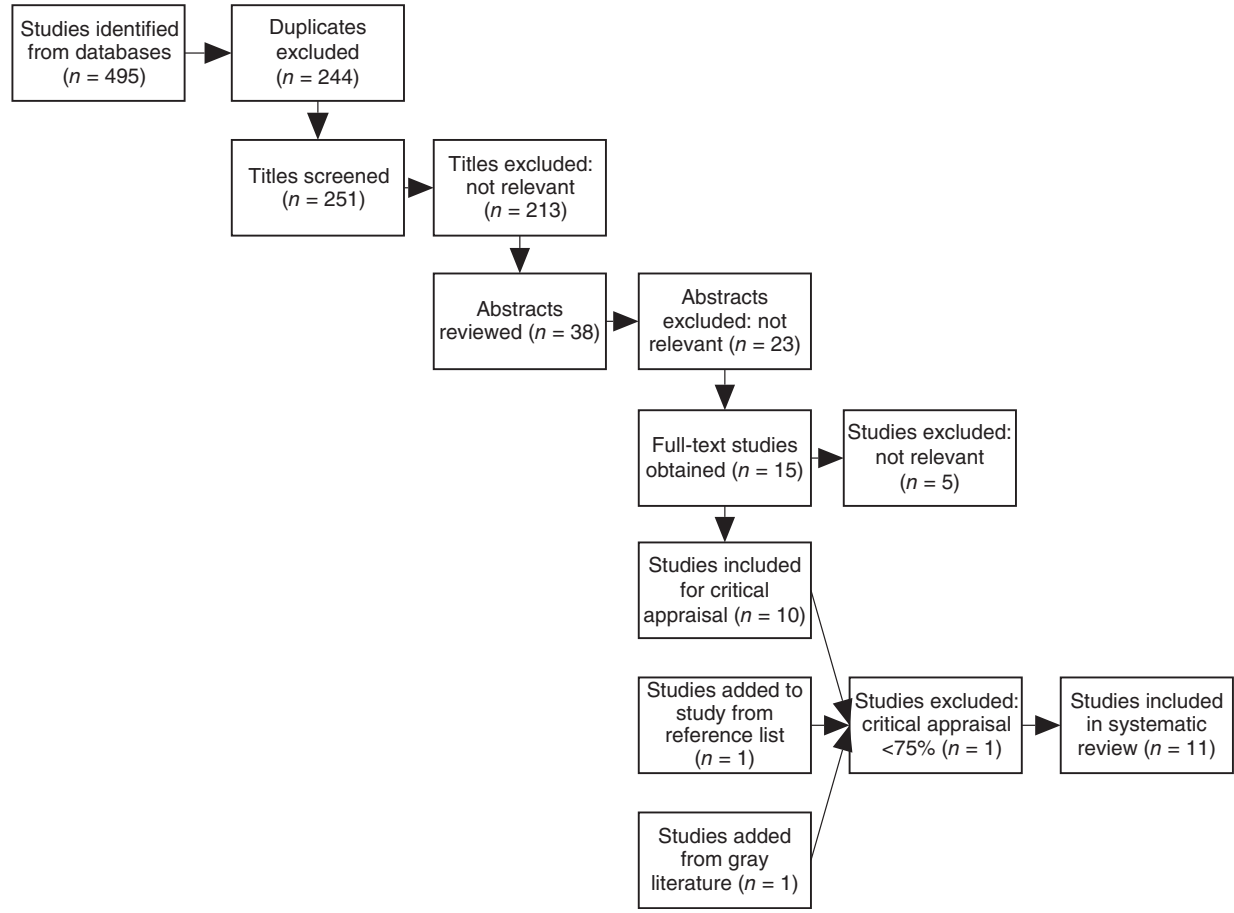

FIGURE 1 Study identification and exclusion process disruptions, the caregiver is destined to develop compassion fatigue (Figley, 2002).

Figley $(1995,2002)$ pioneered the field, and in his model expertly linked empathy with the caregiver's capability to connect with and help a client. He also highlighted the fact that contact with a client requires the investment of personal resources or emotional energy. In addition, he saw the development of compassion fatigue as a process, starting with compassion stress, which if not managed by a sense of satisfaction or disengagement, and with continued exposure to stress, develops into compassion fatigue. One limitation of the model was that empathy was considered to be not only the keystone of helping others, but also the main antecedent in the development of a caregiver's compassion fatigue. Recent research shows, however, that empathy improves not only patient outcomes (Derksen et al., 2013), but also nurse outcomes (Van Dam et al., 2011; Wagaman et al., 2015). Further limitations highlighted by Sabo (2011) include the linear direction of the model, the binary dimension of compassion fatigue, and failure of the model to demonstrate the positive outcomes of helping clients.

The conceptual framework of secondary stress and trauma responses of Valent $(1995,2002)$ is grounded in a stress-process framework. The process begins with exposure to stress; in this case, a client that needs to be helped. The caregiver then cognitively appraises the stressful situation, which evokes survival strategies of rescuing, protecting, and providing for the client. The caregiver's response to these survival strategies might be either adaptive or maladaptive, manifesting in biologic, psychologic, and social dimensions. Adaptive stress responses manifest biologically with increased estrogen, oxytocin, and opioids; psychologically as care, empathy, and devotion; and socially as responsibility, nurturing, and preservation. Maladaptive stress responses manifest biologically with sympathetic and parasympathetic arousal; psychologically as burden, depletion, and self-concern; and socially as resentment, neglect, and rejection.
Strain occurs with a continued maladaptive response to the stress situation, and with continued strain, trauma or compassion fatigue develops. Defenses then set in to minimize the trauma or its repetition, and eventually the person succumbs to illness. Throughout the whole process, endowments resist, and vulnerabilities facilitate, the negative effects of stress.

Valent $(1995,2002)$ was the first person to introduce cognitive appraisal by the caregiver of a stressful situation, and the application of either adaptive or maladaptive stress responses with either positive or negative outcomes for the caregiver. He was also the first person to allude to the balance of resources in either protection against or the development of compassion fatigue. Limitations of this model include failure to explain why some individuals have adaptive stress responses, while others have maladaptive responses. The model also fails to explain the role of cognitive appraisal and the balance of resources in protection against or the development of compassion fatigue.

The professional quality of life model developed by Stamm (2010) describes how the client, work, and personal environments of the caregiver contribute to either a positive (compassion satisfaction) or a negative (compassion fatigue) outcome of helping others. In this model, compassion fatigue consists of two different aspects: burnout and primary or secondary exposure to trauma. The model was the first to introduce the term "compassion satisfaction" as the opposite extreme of compassion fatigue. Furthermore, the model incorporated other avenues of stress, including the work environment and the caregiver's personal environment, coupling the contribution of these environments together with the client's environment to the development either of compassion satisfaction or of compassion fatigue. Limitations to this model are that it describes compassion fatigue as the combination of burnout and secondary traumatic stress, blurring the conceptual boundaries of compassion fatigue. In addition, it fails to describe the actual etiology in the development of either compassion 

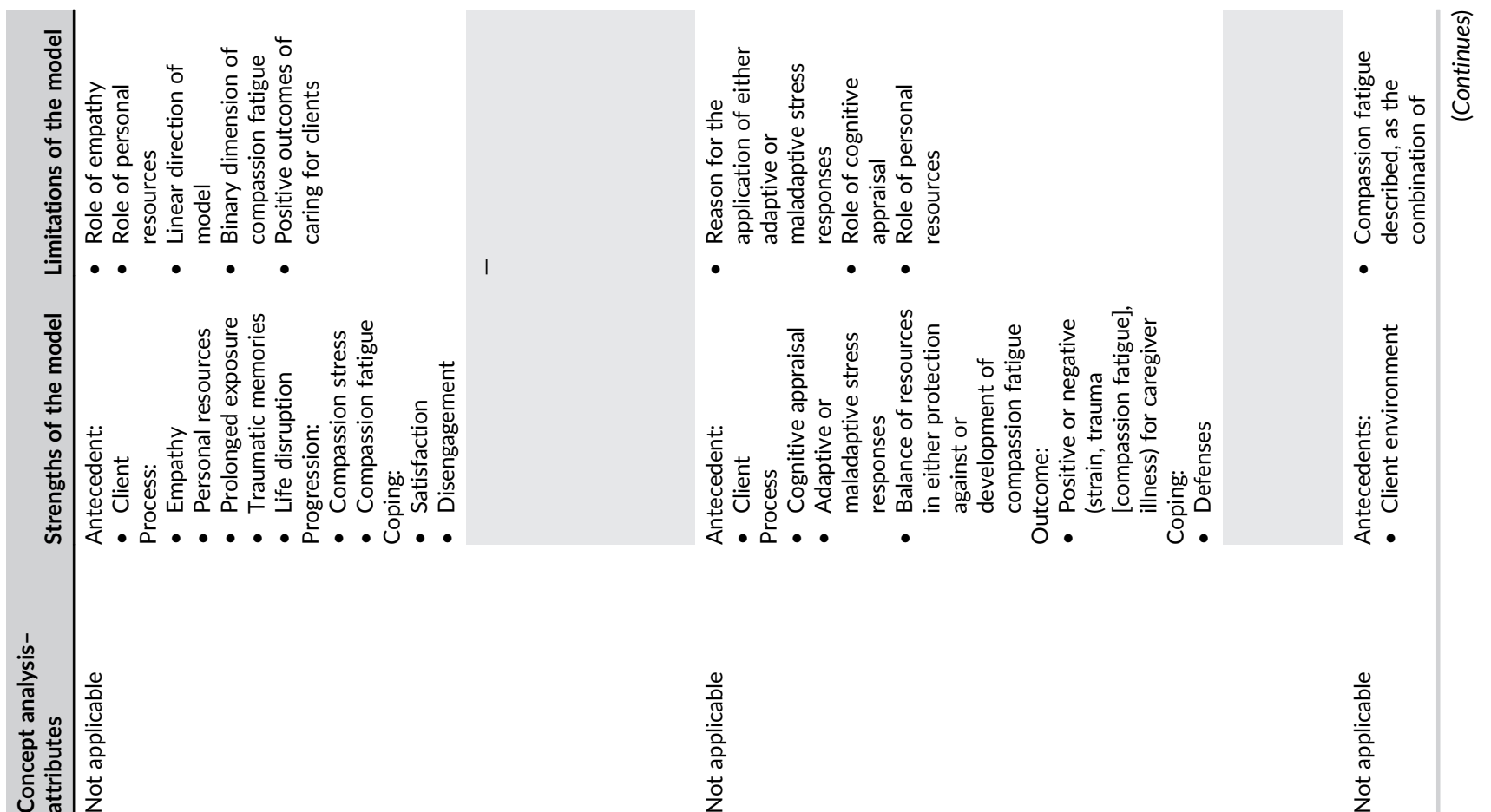

$\frac{0}{\frac{0}{0}}$
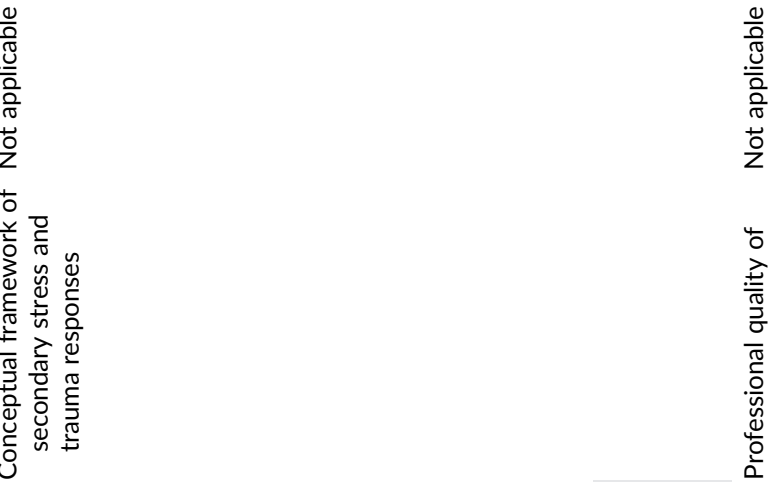

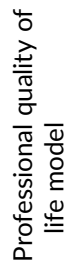

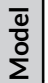

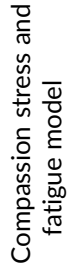
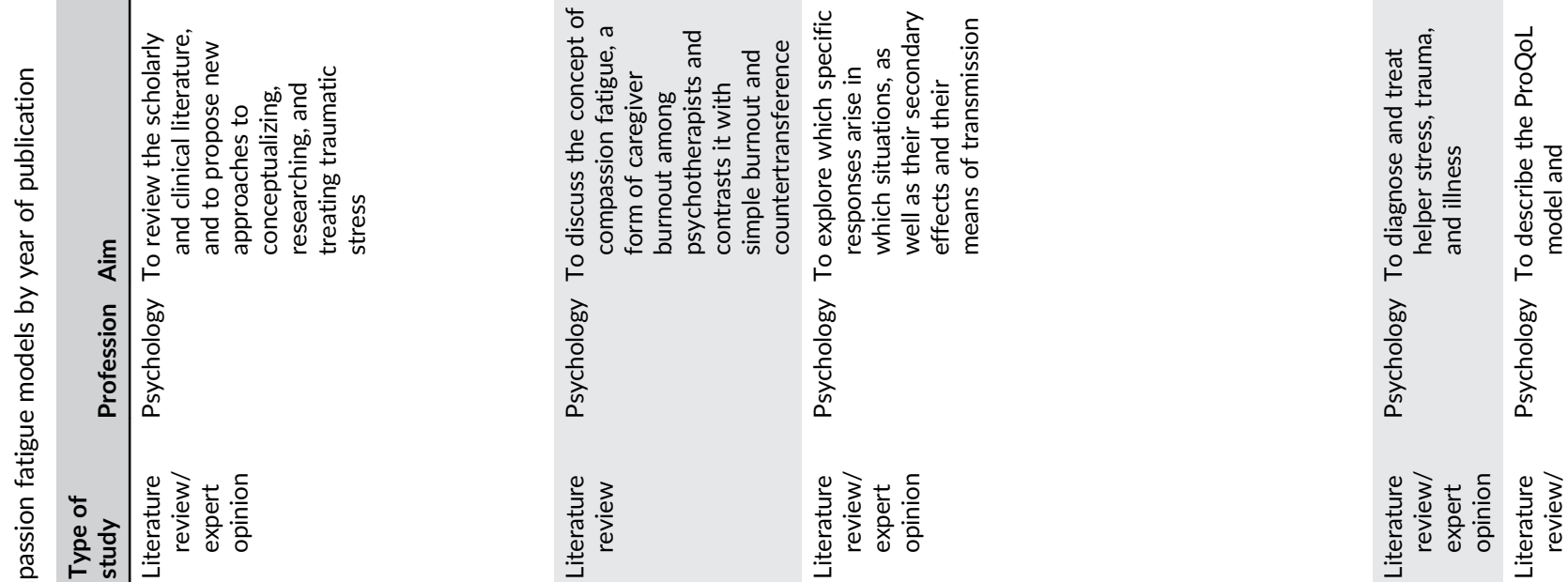

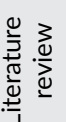

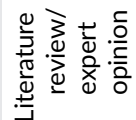

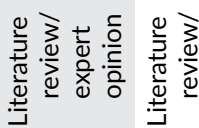
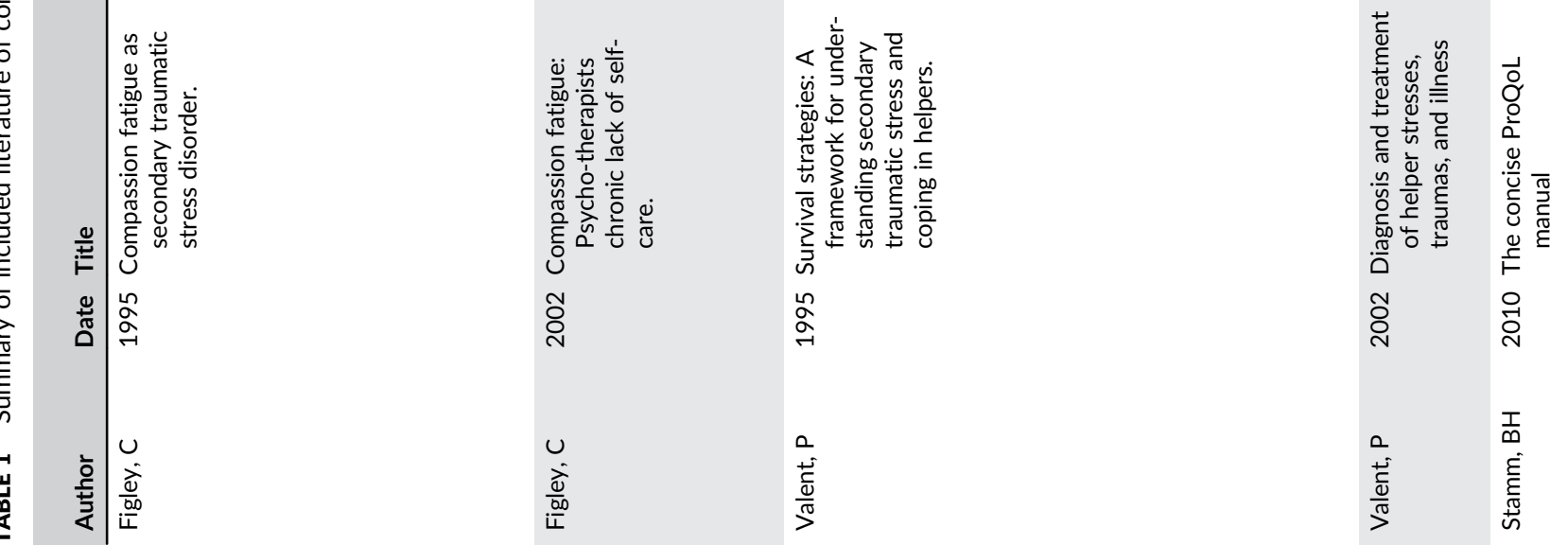


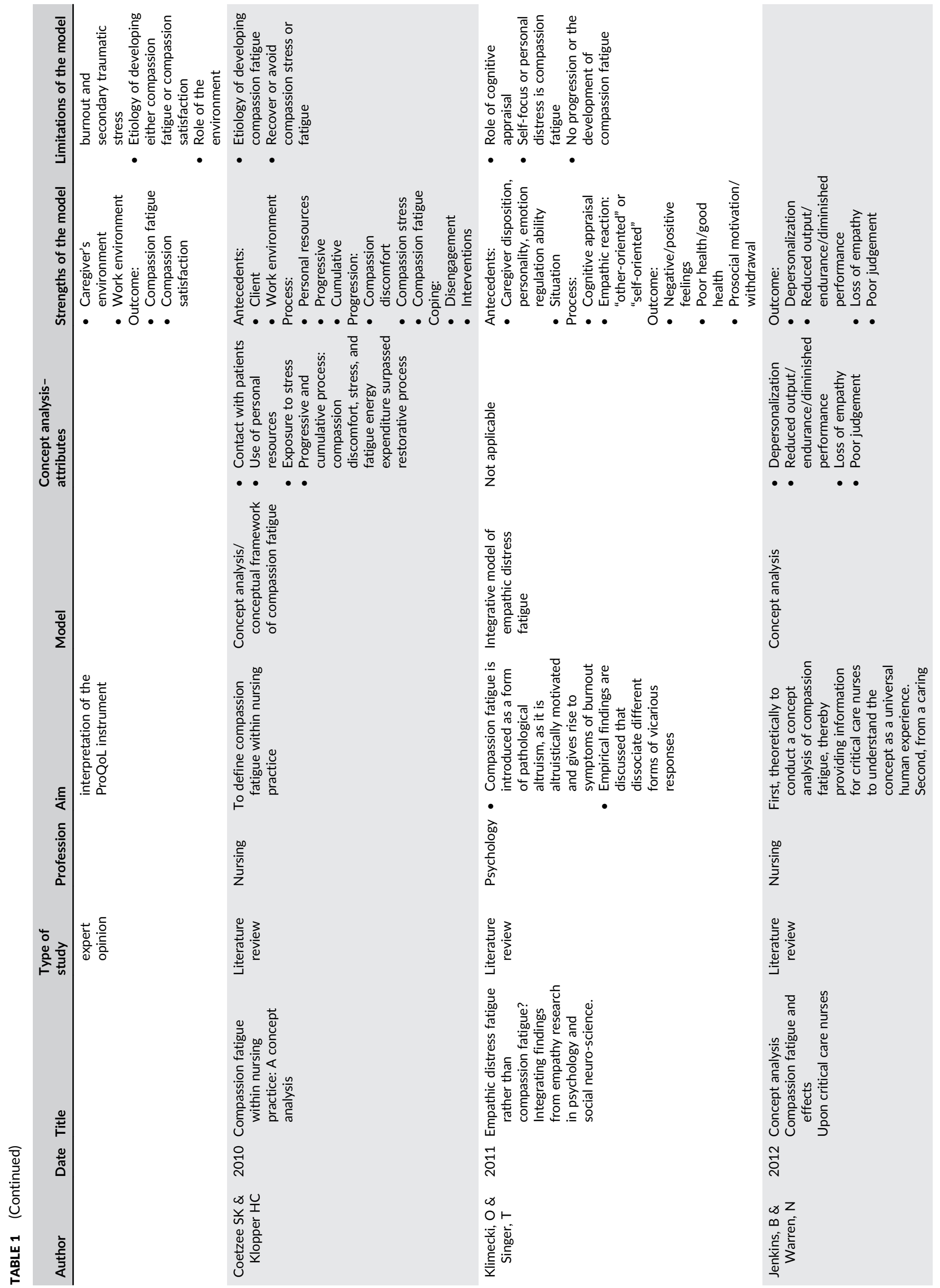




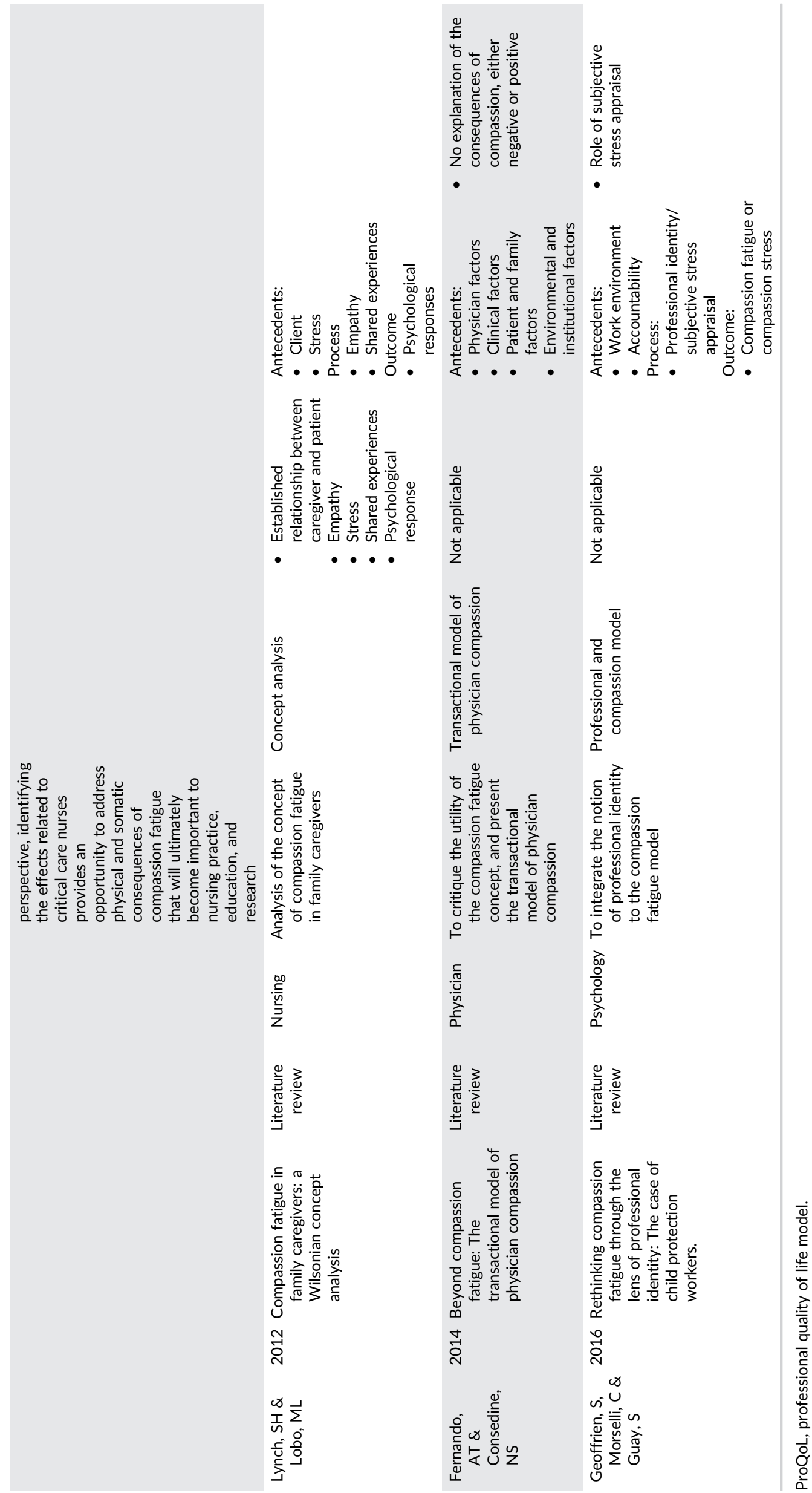


fatigue or compassion satisfaction, and does not explain the role of the environments in protection against or the development of compassion fatigue.

The conceptual framework of compassion fatigue outlined by Coetzee and Klopper (2010) was based on an integrative literature review, and described the development of compassion fatigue as being progressive and cumulative. Risk factors were identified as having contact with patients, use of resources, and environmental stress. Prolonged, continuous, and intense contact with these risk factors resulted in the caregiver developing compassion discomfort, followed, if no intervention was applied at this point, by compassion stress. In order to cope with compassion stress, caregivers distance and isolate themselves from their clients, and meaningful and purposeful interaction between the caregiver and client is lost, resulting in compassion fatigue. Unique to this conceptual framework is the addition of compassion discomfort as an entry point in the development of compassion fatigue. A limitation to this conceptual framework is that it does not explain the etiology of compassion fatigue, and although we indicate that interventions can be applied at the level of compassion discomfort, the framework does not indicate how a caregiver can recover from or avoid compassion stress and fatigue.

Klimecki and Singer (2011) provided a new perspective of compassion fatigue by applying social and developmental psychology and social neuroscience research to develop an integrative model of compassion fatigue. In their model, empathy is also central to the provision of health care, but in this model, the consequence of empathy can take on two paths, either an "other-oriented focus" or compassion, which results in prosocial motivation, positive feelings, and good health, or a "self-oriented focus" or empathic/personal distress, which results in withdrawal, poor health, and negative feelings. Klimecki and Singer (2011) highlight that it is the ability of the caregiver to differentiate between themselves and the patient's suffering that leads to an "other-oriented focus," while identifying with the patient's suffering as if it were their own leads to a "selforiented focus." The empathic reaction applied by the caregiver depends on his/her disposition, personality, emotion regulation ability, and the situation. This model was the first to highlight that empathy can take two different paths based on personal and situational factors. Critique of this model would be that the authors imply that a self-oriented focus or personal distress is compassion fatigue, thus there is no progression or development of compassion fatigue. Consequently, in every caregiving interaction, the person will apply either a self-oriented focus or other-oriented focus, based on personal and situational factors, and experience either compassion fatigue or compassion satisfaction as a result. This model is then in contradiction with the other models discussed. Furthermore, the process alludes to cognitive appraisal in the application of an empathic reaction, but its role in the model is not clear.

In 2012, two further concept analyses were conducted on compassion fatigue, specifically among critical care nurses (Jenkins \& Warren, 2012) and family caregivers (Lynch \& Lobo, 2012). Jenkins and Warren (2012) focused more specifically on the outcome attributes of compassion fatigue, while Lynch and Lobo (2012) highlighted similar antecedent, process, and outcome attributes as the previous models discussed.
The transactional model of physician compassion proposed by Fernando and Consedine (2014) adopted a wellness approach and developed a model that captured the dynamic and transactional nature of providing compassion, together with the factors involved in the enhancement and/or mitigation of compassion in clinical care. These factors were identified as physician factors, clinical factors, patient factors, and family factors within the overarching environmental and institutional factors that explain a caregiver's ability to provide compassion. This transactional model of physician compassion uniquely identified clinical factors (the degree to which a physician might consciously or unconsciously consider a patient "responsible" for their condition, the complexity of the situation, and the physician's expertise) as additional stressors or antecedents of compassion fatigue. Limitations of this model are that it focused only on the antecedents that enhance and/or mitigate compassion, and did not explain the consequences of compassion, either positive or negative.

Finally, the professional and compassion module (Geoffrion et al., 2015) identifies work-related stressors as primary traumatic stress, STS, vicarious traumatic stress, and accountability stress, and includes professional identity as a subjective interpretive framework that interprets and gives meaning to work-related external stressors and modulates compassion fatigue. This model uniquely focuses on the stress engendered by accountability. It also further builds on aspects of Valent's (1995, 2002) model, incorporating subjective stress appraisal, but further shows that this impacts on the development of either compassion satisfaction or compassion fatigue. Limitations of this model are that the exact role of subjective stress appraisal on the development of either compassion fatigue or compassion satisfaction is unclear.

\section{4 | DISCUSSION}

It is apparent from the results that these models have enhanced the theoretical understanding of compassion fatigue. The models are congruent with regard to the positive and negative outcomes of caring for clients (Coetzee \& Klopper, 2010; Figley, 1995, 2002; Geoffrion et al., 2015; Jenkins \& Warren, 2012; Klimecki \& Singer, 2011; Lynch \& Lobo, 2012; Stamm, 2010; Valent, 1995, 2002), as well as the antecedents of compassion fatigue. These antecedents are identified as clients or client factors (Coetzee \& Klopper, 2010; Fernando \& Consedine, 2014; Figley, 1995, 2002; Lynch \& Lobo, 2012; Stamm, 2010; Valent, 1995, 2002), the work environment (Coetzee \& Klopper, 2010; Fernando \& Consedine, 2014; Geoffrion et al., 2015; Klimecki \& Singer, 2011; Lynch \& Lobo, 2012; Stamm, 2010), caregiver factors (Fernando \& Consedine, 2014; Geoffrion et al., 2015; Klimecki \& Singer, 2011; Stamm, 2010), and clinical factors (Fernando \& Consedine, 2014).

The models are more unclear when it comes to the process or etiology of compassion fatigue, but the main aspects alluded to are the balance of resources (Coetzee \& Klopper, 2010; Figley, 1995, 2002; Klimecki \& Singer, 2011; Lynch \& Lobo, 2012; Valent, 1995, 2002), the use of empathy (Coetzee \& Klopper, 2010; Figley, 1995, 2002; Klimecki \& Singer, 2011; Lynch \& Lobo, 2012), and stress 
appraisal (Geoffrion et al., 2015; Klimecki \& Singer, 2011; Valent, $1995,2002)$ in the development of either compassion fatigue or compassion satisfaction.

We aim to build further on these models and explain the process or etiology of compassion fatigue through the application of the conservation of resources (COR) theory (Hobfoll, 1989, 1998) to explain the balance of resources, and social neuroscience of empathy research (Decety \& Lamm, 2009; Gonzalez-Liencres et al., 2013) to explain the use of empathy and stress appraisal.

\section{1 | Conservation of resources theory}

To explain how the balance of resources influences the etiology of either compassion fatigue or compassion satisfaction, the COR theory (Hobfoll, 1989, 1998) was applied, as it is an integrated model of stress, and comprises of several stress theories.

The central tenet of COR theory is that because people value resources, they work to acquire resources they do not have, they retain those resources that they possess, they protect resources that are threatened, and they foster resources by ensuring that their resources can be put to their best use (Hobfoll, 1989, 1998).

Following this tenet, COR theory includes two major principles and several secondary corollaries (Hobfoll, 1998). The first and most important principle is that "resource loss is disproportionately more salient than is resource gain" (p. 62). The second principle states that "people must invest resources in order to protect against resource loss, recover from losses, and gain resources" (p. 73). The four corollaries are:

1. "Those with greater resources are less vulnerable to resource loss and more capable of orchestrating resource gain. Conversely, those with fewer resources are more vulnerable to resource loss and less capable of achieving resource gain" (p. 80),

2. "Those who lack resources are more vulnerable to resource loss, and initial loss begets future loss" (p. 81),

3. "Those who possess resources are both more capable of gain, and resource gain begets further gain" (p. 82), and

4. "Those who lack resources are likely to adopt a defensive posture or guard their resources" (p. 83)

These principles and corollaries will be applied to the compassion fatigue model (CFM) to show how the balance of resources influences the etiology of either compassion fatigue or compassion satisfaction.

\subsection{Social neuroscience of empathy}

To explain how empathy and stress appraisal influences the etiology of either compassion fatigue or compassion satisfaction, social neuroscience of empathy research was applied (Decety \& Lamm, 2009; Gonzalez-Liencres et al., 2013).

Empathy is the ability of the caregiver to perceive, imagine, or infer the client's suffering, sorrow, or pain, and express motivation to improve the patient's experience, with full awareness of the distinction between themselves and the patient (Decety \& Lamm, 2009;
Gonzalez-Liencres et al., 2013). Social neuroscience has shown that specific neural structures, namely the bilateral anterior insular cortex and medial/anterior cingulate cortex, are associated with empathy for pain, and this overlaps with activation during directly experienced pain (Lamm, Decety, \& Singer, 2011). Studies using functional neuroimaging demonstrate that the object of one's focus (other or self) has different emotional and behavioral consequences, and so do the processing modes (experiential or propositional) applied (Decety, Smith, Norman, \& Halpern, 2014).

With regard to the object of focus, other or self, both allow the caregiver to acknowledge and feel similar feelings to the patient. However, in the other focus, the caregiver understands that the patient's feelings are different from their own, and they can separate their feelings from those of the patient. Conversely, in self-focus, the caregiver experiences the patient's feelings as if these were their own, confusing the patient's feelings with their own (Decety \& Lamm, 2009; Gleichgerrcht \& Decety, 2013). Furthermore, neuroscience suggests that there are two modes of processing information in empathy: the experiential or propositional processing modes. The experiential processing mode is usually an involuntary response or bottom-up approach, while the propositional processing mode is usually a voluntary response or top-down approach (Decety et al., 2014).

The object of focus (other or self) and the processing mode (experiential or propositional) of each focus will be applied to the CFM to show how empathy and stress appraisal influence the etiology of either compassion fatigue or compassion satisfaction.

\section{3 | Compassion fatigue model}

The CFM developed in this review is based on previous models of compassion fatigue (Coetzee \& Klopper, 2010; Fernando \& Consedine, 2014; Figley, 1995, 2002; Geoffrion et al., 2015; Jenkins \& Warren, 2012; Klimecki \& Singer, 2011; Lynch \& Lobo, 2012; Valent, 1995, 2002), on COR theory (Hobfoll, 1989, 1998) and on social neuroscience of empathy research (Decety \& Lamm, 2009; GonzalezLiencres et al., 2013), and extends knowledge in these areas.

The CFM is applied to one cycle of caregiver-patient interaction (Figure 2).

\subsection{1 | Resources}

As explained in the discussion about COR theory, every caregiver has their own balance of resources. These resources include object, conditional, personal, and energy resources (Hobfoll, 1998). Object resources are those resources that have a physical presence and are valued for their function or status (Hobfoll, 1998), such as infrastructure and adequate staffing. Condition resources are structures or states that lay a foundation for access to or possession of other resources, such as spirituality or being healthy (Hobfoll, 1998). Personal resources are most frequently acquired through learning and result from modeling, education, and role adoption. Personal resources include both personal skills and traits. Personal skills encompass, for example, occupational skills and leadership ability, while personal traits include self-esteem and resilience (Hobfoll, 1998). Energy resources derive their value from their ability to be 


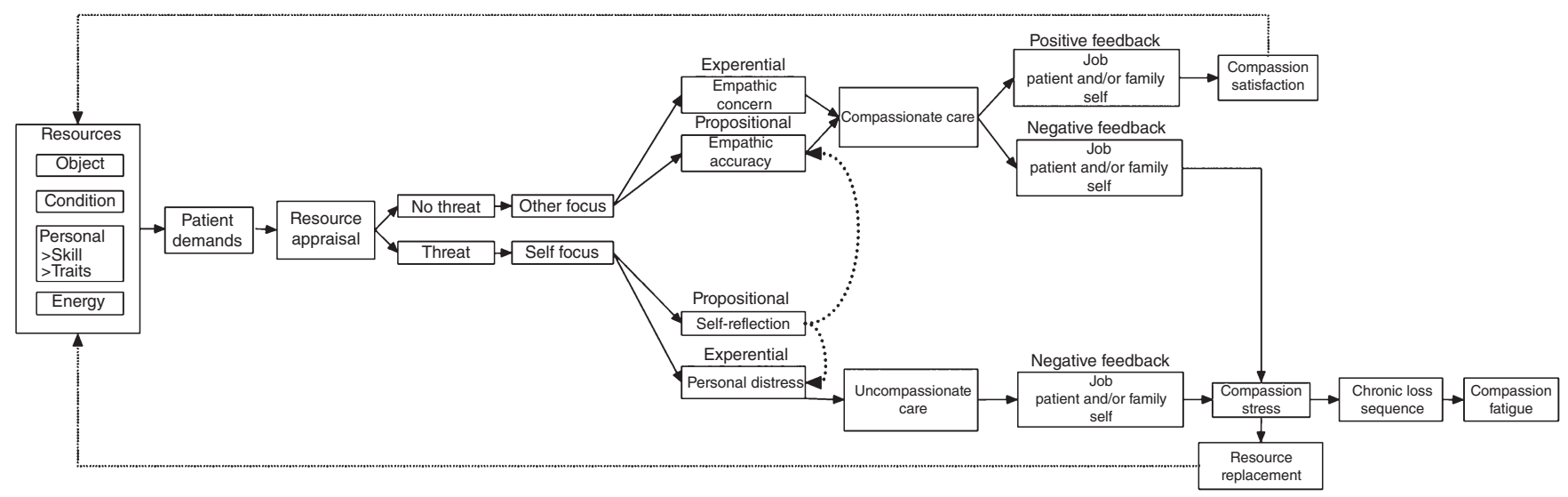

FIGURE 2 Compassion fatigue model

exchanged for resources in the other three categories. Energy resources include actual energy, time, and knowledge (Hobfoll, 1998).

As an example, a caregiver's resource balance might be well resourced, in that there is adequate resources in the ward (object resource), he/she is happily married (condition resource), is well educated (personal skill), and optimistic (personal trait), with sufficient physical energy (energy resource). Or the caregiver's resource balance might be impoverished, in that he/she is bullied at work (object resource), is a single parent (condition resource), has no conflict management skills (personal skill), and is neurotic (personal trait), with inadequate emotional energy (energy resource). A caregiver could have varying levels of different resources, but the sum of these resources will make him/her either well resourced or resource impoverished.

\subsection{2 | Resource appraisal}

Faced with a patient's demands (e.g. severity of the illness and/or level of dependence), the caregiver will appraise their resource balance in relation to the demands. In the case of a caregiver who is well resourced, they will not experience the patient's needs as a threat to their resources. In fact, the caregiver will view it as an opportunity to gain resources (COR, corollary 1 ) and will have an empathic focus on the patient (other focus). Alternatively, the caregiver might be resource impoverished and feel that the patient's needs pose a threat (Joireman, Parrott, \& Hammersla, 2002) to their own resources (COR, corollary 1). The caregiver would thus opt to retain their resources and to have an empathic focus on themselves (self-focus). Either way, the caregiver would be investing resources in order to protect against resource loss, recover from losses, or gain resources (COR, principle 2).

\subsection{3 | Processing modes}

The object of one's focus (other or self) has different emotional and behavioral consequences, but so do the processing modes that the caregiver might adopt. The first mode is experiential processing (Rameson \& Lieberman, 2009), which is also known as the mirror system, motor and affective resonance, or emotional sharing (Decety \& Lamm, 2009). The second is propositional processing (Rameson \& Lieberman, 2009), which is also known as the mentalizing system, metacognition, or theory of mind (Decety \& Lamm, 2009). Experiential processing is an involuntary, automatic affective reaction, which matches an affective experience between the caregiver and the patient, while propositional processing is a voluntary, controlled cognitive process that enables the caregiver to adopt the perspective of others (Rameson \& Lieberman, 2009).

\subsection{4 $\mid$ Other focus}

An other focus allows the caregiver to acknowledge or empathize with the patient, in that similar feelings are felt by the caregiver and the patient, while understanding that those feelings are different from the caregiver's own (no confusion between the individuals) (Decety \& Lamm, 2009; Gleichgerrcht \& Decety, 2013). Otherfocused experiential processing, termed "empathic concern", is when the caregiver is able to enter into the patient's experience of suffering or pain, and experience the situation as if it were happening to him/herself, in a very automatic way, but with self-other distinction (Gleichgerrcht \& Decety, 2013; Rameson \& Lieberman, 2009). This type of processing often results in immediate physical engagement (Rameson \& Lieberman, 2009). Other-focused propositional processing, termed "empathic accuracy", employs controlled reasoning that infers the patient's thoughts and feelings, while understanding that they differ from the caregiver's own thoughts and feelings (Gleichgerrcht \& Decety, 2013; Rameson \& Lieberman, 2009). This type of process often results in thinking about solutions to problems that will change the patient's situation. Whatever other-focused processing mode is applied, the result is a connection with the patient and a desire to alleviate or remove the patient's suffering or pain.

With this connection and desire to help the patient, the caregiver engages with the patient and responds to the patient's need, investing their personal resources to provide compassionate care. Having invested their personal resources, and perceiving they have compassionately cared for the patient, the caregiver will expect some resource gain. The resource gain comes from positive feedback from their own: biologic, psychologic, and social dimensions; from the patient/family: positive patient outcome, praise from patient and/or family; and/or from the job: praise from colleagues or managers, and work control. Neuroimaging studies have demonstrated that compassionate action is associated with the activation of areas in the brain associated with reward (Jensen et al., 2014). Furthermore, Morrison and Korol (2014) found that acknowledgment, rewards, and positive 
energy enable the caregiver to feel connected to the patient, the job, and colleagues. This positive feedback results in resource gain (compassion satisfaction), and the caregiver will be even more motivated to gain resources in the next cycle (COR, corollary 2). GonzalezLiencres et al. (2013) reinforce this corollary, stating that empathy triggers cooperation, which in turn increases reciprocity that once again positively impacts on empathy. Furthermore, Gleichgerrcht and Decety (2013) have shown in a study with 7584 board-certified practicing physicians that both empathic concern and empathic accuracy are closely associated with compassion satisfaction.

However, the caregiver might have invested resources and compassionately cared for the patient, but is instead faced with a negative outcome, as in the case of a health complication or death, which results in negative feedback from their own biologic, psychologic, and social dimensions, from the patient/family (negative patient outcome, no recognition, complaints), or from the job (no recognition, criticism, complaints). The consequence is a loss of resources. Siegrest (1996) states that if there is a high level of effort-reward imbalance, meaning there is a high degree of effort (resource investment) with few rewards (resource gain), this will be a highly stress-generating condition (compassion stress). At the point of compassion stress, the caregiver is still able to apply resource replacement (e.g. debriefing, supervised practice). However, according to COR theory, the resource loss is disproportionately more salient than the resource gain (COR, principle 1), and the caregiver will be more motivated to protect him/herself against resource loss in the next cycle.

\subsection{5 | Self-focus}

With self-focus, similar feelings are felt by the caregiver and the patient, but in this instance there is confusion (no distinction) between the self and other (Decety \& Lamm, 2009). According to Rameson and Lieberman (2009), in this state of self-focus, the caregiver will also process empathy either experientially or propositionally.

Self-focused propositional processing will result in self-reflection, which is a controlled cognitive evaluation of the caregiver's own thoughts and feelings about the patient's situation (Rameson \& Lieberman, 2009). Self-reflection is associated with openness to experience (Joireman et al., 2002), and often results from social rules, experience, training, and knowledge (Rameson \& Lieberman, 2009; Thomas, 2013). This allows the caregiver to think about their "own thoughts and feelings, recognize biases, monitor errors in thinking, and engage in ongoing reappraisal" (Thomas, 2013, p. 370). Such thoughts and feelings allow the caregiver to consider perspectives that depart from his/her own (Joireman et al., 2002), and possibly gain an other focus, employing controlled reasoning, separating from the situation (empathic accuracy) (Gleichgerrcht \& Decety, 2013; Rameson \& Lieberman, 2009). However, it is possible that after selfreflection, the caregiver might revert to personal distress.

Self-focused experiential processing results in personal distress. Personal distress is an aversive emotional reaction to another's plight, which motivates withdrawal or avoidance (Lamm et al., 2011). In this state, the caregiver will distance from the unpleasant stimulus: the patient. No longer acknowledging or empathizing with the patient, the caregiver is unable to connect with the patient and cannot accurately ascertain the patient's needs (Eisenberg \& Eggum, 2009; Rameson \& Lieberman, 2009), providing "just the basics"' of care (Austin, Goble, Leier, \& Byrne, 2009, p. 204). The patient, sensing the caregiver's stress, responds in a similar fashion by distancing from the caregiver (Austin et al., 2009). Research has shown, although not in the medical field, that parents with higher levels of personal distress engage in more negative verbal and expressive behavior toward their children (Valiente et al., 2004) and are also more likely to physically abuse their children (De Paúl, Pérez-Albéniz, Guibert, Asla, \& Ormaechea, 2008). These findings might apply to the patient-caregiver context in some instances (McMahon et al., 2014). As a result, the patient receives uncompassionate care and the caregiver receives negative feedback from one or more of the following: (i) from themselves: people with personal distress are more likely to ruminate and have lower self-esteem (Joireman et al., 2002), be depressed (Thoma et al., 2011), with no release of neuropeptides (Gleichgerrcht \& Decety, 2013); (ii) from the patient/family: negative patient outcome, no recognition, complaints; and (iii) from the job: no recognition, criticism, complaints, gossip. The consequence is a further loss of resources (compassion stress). Morrison and Korol (2014) found that not receiving positive feedback or rewards resulted in disconnectedness from patients, the job, and colleagues. The caregiver should replace resources at this point, or the cycle will continue to repeat itself (COR, corollary 3). When a chronic loss sequence occurs, the caregiver will adopt a defensive posture and socially and emotionally isolate from resource loss (COR, corollary 3), and experience compassion fatigue. Gleichgerrcht and Decety (2013) and Thomas (2013) found that personal distress was associated with compassion fatigue.

\section{4 | Implications for nursing and midwifery practice}

The CFM shows that a poor-resourced nurse is more likely to experience compassion fatigue. Although most resources relate to an individual level (conditional, personal, and energy resources), the practice environment (object resource) is an external resource that can be addressed by policies governing the healthcare sector and the healthcare facility and units.

It is well documented that a positive practice environment results in better nurse and patient outcomes (Aiken et al., 2012), and a systematic review conducted by (Lambrou \& Merkouris, 2014) found that the most important aspects of the practice environment influencing job satisfaction are the manager's ability, leadership, and support of nurses, and nurses' involvement in decision-making. Thus, the healthcare sector and healthcare facilities should invest resources in the training, mentoring, and support of nurse managers in healthcare facilities and units. Furthermore, nurse managers and nurses at all levels should be involved in policy development and decisionmaking in the healthcare sector and healthcare facilities when such decisions will influence the nursing profession and patient care.

Resource replacement can also be addressed by the healthcare sector and by healthcare facilities and units through continuous professional development and in-service training focused on selfenrichment education and life-skills training. Moreover, the facility or 
unit manager can be influential in orchestrating resource replacement initiatives, such as support groups and supervised practice.

However, it would seem that the single most important intervention for policy and practice is that the nurse should work in an environment of positive feedback, where he/she is acknowledged, rewarded, and praised. Innovative branding and public awareness campaigns nationally and in healthcare facilities and units will contribute to a positive image and appreciation of nursing. Furthermore, healthcare facilities and units should have nurse employee reward and recognition systems in place, as well as policies to protect nurses from collegial, patient, and family incivility and bullying.

Finally, with compassion-related training and education in undergraduate and postgraduate education programs, nurses should be able to develop strategies to reduce self-focused personal distress and manifest greater empathy toward patients.

\section{5 | CONCLUSION}

The CFM developed in this review built on the strengths of previous models of compassion fatigue (Coetzee \& Klopper, 2010; Fernando \& Consedine, 2014; Figley, 1995, 2002; Geoffrion et al., 2015; Jenkins \& Warren, 2012; Klimecki \& Singer, 2011; Lynch \& Lobo, 2012; Stamm, 2010; Valent, 1995, 2002), and addressed limitations through the application of COR theory (Hobfoll, 1989, 1998) and social neuroscience of empathy research (Decety \& Lamm, 2009; Gonzalez-Liencres et al., 2013). The CFM explains the role of the balance of resources, empathy, and stress appraisal in the development of either compassion fatigue or compassion satisfaction. The CFM demonstrates that it is not empathy that puts nurses at risk of developing compassion fatigue, but rather a lack of resources, an absence of positive feedback, and the response of personal distress. It is timely for nurse leaders to act on the findings of the CFM so as to improve the retention of a compassionate and committed nurse workforce.

\section{ACKNOWLEDGMENTS}

This study was made possible through the international postdoctoral fellowship in nursing, sponsored jointly by the International Network for Doctoral Education in Nursing and Sigma Theta Tau International. This work is based on the research supported by the National Research Foundation of South Africa (grant no. 84160). Any opinion, finding and conclusion or recommendation expressed in this material is that of the author(s) and the NRF does not accept any liability in this regard.

\section{AUTHOR CONTRIBUTIONS}

Study design: S.K.C. and H.K.S.L.

Data collection: S.K.C.

Data analysis: S.K.C.

Manuscript writing and revisions for important intellectual content: S.K.C. and H.K.S.L.

\section{ORCID}

Siedine K. Coetzee (D) http://orcid.org/0000-0002-6042-0926

\section{REFERENCES}

Aiken, L. H., Sermeus, W., Van den Heede, K., Sloane, D. M., Busse, R., McKee, M., ... Kutney-Lee, A. (2012). Patient safety, satisfaction, and quality of hospital care: cross sectional surveys of nurses and patients in 12 countries in Europe and the United States. BMJ (Clinical Research Ed.), 344, e1717. https://doi.org/10.1136/bmj.e1717.

Austin, W., Goble, E., Leier, B., \& Byrne, P. (2009). Compassion fatigue: The experience of nurses. Ethics \& Social Welfare, 3(2), 195-214. https://doi.org/10.1080/17496530902951988.

Coetzee, S. K., \& Klopper, H. C. (2010). Compassion fatigue within nursing practice: A concept analysis. Nursing and Health Sciences, 12(2), 235-243. https://doi.org/10.1111/j.1442-2018.2010.00526.x.

De Paúl, J., Pérez-Albéniz, A., Guibert, M., Asla, N., \& Ormaechea, A. (2008). Dispositional empathy in neglectful mothers and mothers at high risk for child physical abuse. Journal of Interpersonal Violence, 23(5), 670-684. https://doi.org/10.1177/0886260507313532.

Decety, J., \& Lamm, C. (2009). Empathy versus personal distress: Recent evidence from social neuroscience. In J. Decety \& W. Ickes (Eds.), The social neuroscience of empathy (pp. 199-213). Cambridge, MA: The MIT Press.

Decety, J., Smith, K., Norman, G., \& Halpern, J. (2014). A social neuroscience perspective on clinical empathy. World Psychiatry, 13(3), 233-237.

Derksen, F., Bensing, J., \& Lagro-Janssen, A. (2013). Effectiveness of empathy in general practice: A systematic review. British Journal of General Practice, 63(606), 76-84. https://doi.org/10.3399/bjgp13X660814.

Eisenberg, N., \& Eggum, N. (2009). Empathic responding: Sympathy and personal distress. In J. Decety \& W. Wickes (Eds.), The social neuroscience of empathy (pp. 71-84). Cambridge, MA: The MIT Press.

Fernando, A. T., \& Consedine, N. S. (2014). Beyond compassion fatigue: The transactional model of physician compassion. Journal of Pain and Symptom Management, 48(2), 289-298. https://doi.org/10.1016/j. jpainsymman.2013.09.014.

Figley, C. (1995). Compassion fatigue: Coping with STS disorder in those who treat the traumatized. London, England: Brunner-Routledge.

Figley, C. (2002). Compassion fatigue: Psychotherapists' chronic lack of self care. Journal of Clinical Psychology, 58(11), 1433-1441.

Geoffrion, S., Morselli, C., \& Guay, S. (2015). Rethinking compassion fatigue through the lens of professional identity: The case of childprotection workers. Trauma, Violence \& Abuse, 17(3), 270-283. https://doi.org/10.1177/1524838015584362.

Gleichgerrcht, E., \& Decety, J. (2013). Empathy in clinical practice: How individual dispositions, gender, and experience moderate empathic concern, burnout, and emotional distress in physicians. PloS One, 8(4), e61526. https://doi.org/10.1371/journal.pone.0061526.

Gonzalez-Liencres, C., Shamay-Tsoory, S. G., \& Brune, M. (2013). Towards a neuroscience of empathy: Ontogeny, phylogeny, brain mechanisms, context and psychopathology. Neuroscience and Biobehavioral Reviews, 37(8), 1537-1548. https://doi.org/10.1016/j.neubiorev.2013.05.001.

Hobfoll, S. E. (1989). Conservation of resources. A new attempt at conceptualizing stress. The American Psychologist, 44(3), 513-524. https:// doi.org/10.1037/0003-066X.44.3.513.

Hobfoll, S. E. (1998). Stress, culture and community: The psychology and philosophy of stress. London, England: Plenum Press.

Jenkins, B., \& Warren, N. (2012). Concept analysis: Compassion fatigue and effects upon critical care nurses. Critical Care Nurse Quarterly, 35(4), 388-395.

Jensen, K. B., Petrovic, P., Kerr, C. E., Kirsch, I., Raicek, J., Cheetham, A., ... Kaptchuk, T. J. (2014). Sharing pain and relief: Neural correlates of physicians during treatment of patients. Molecular Psychiatry, 19(3), 392-398. https://doi.org/10.1038/mp.2012.195.

Joinson, C. (1992). Coping with compassion fatique. Nursing, 22(4), 116-118. https://doi.org/10.1097/00152193-199204000-00035.

Joireman, J. A., Parrott, L., \& Hammersla, J. (2002). Empathy and the selfabsorption paradox: Support for the distinction between self- 
rumination and self-reflection. Self and Identity, 1(1), 53-65. https:// doi.org/10.1080/152988602317232803.

Klimecki, O., \& Singer, T. (2011). Empathic distress fatigue rather than compassion fatigue? Integrating findings from empathy research in psychology and social neuroscience. In B. Oakley, A. Knafo, G. Mahavana, \& D. Wilson (Eds.), Pathological altruism (pp. 368-383). New York, NY: Oxford University Press.

Lambrou, P., \& Merkouris, A. (2014). Nurses' perceptions of their professional practice environment in relation to job satisfaction: A review of quantitative studies. Health Science Journal, 8(3), 298-317.

Lamm, C., Decety, J., \& Singer, T. (2011). Meta-analytic evidence for common and distinct neural networks associated with directly experienced pain and empathy for pain. Neurolmage, 54(3), 2492-2502. https:// doi.org/10.1016/j.neuroimage.2010.10.014.

Ledoux, K. (2015). Understanding compassion fatigue: Understanding compassion. Journal of Advanced Nursing, 71(9), 2041-2050. https:// doi.org/10.1111/jan.12686.

Lynch, S. H., \& Lobo, M. L. (2012). Compassion fatigue in family caregivers: A Wilsonian concept analysis. Journal of Advanced Nursing, 68(9), 2125-2134. https://doi.org/10.1111/j.1365-2648.2012.05985.x.

McMahon, S. A., George, A. S., Chebet, J. J., Mosha, I. H., Mpembeni, R. N., \& Winch, P. J. (2014). Experiences of and responses to disrespectful maternity care and abuse during childbirth; a qualitative study with women and men in Morogoro Region, Tanzania. BMC Pregnancy and Childbirth, 14(1), 268. https://doi.org/10.1186/ 1471-2393-14-268.

Morrison, K. B., \& Korol, S. A. (2014). Nurses' perceived and actual caregiving roles: Identifying factors that can contribute to job satisfaction. Journal of Clinical Nursing, 23(23-24), 3468-3477. https://doi.org/10. 1111/jocn.12597.

Rameson, L., \& Lieberman, M. (2009). Empathy: A social cognitive neuroscience approach. Social and Personality Psychology Compass, 3(1), 94-110.

Sabo, B. (2006). Compassion fatigue and nursing work: Can we accurately capture the consequences of caring work? International Journal of Nursing Practice, 12(3), 136-142. https://doi.org/10.1111/j.1440172X.2006.00562.x.

Sabo, B. (2011). Reflecting on the Concept of Compassion Fatigue. Online Journal of Issues in Nursing, 16(1). https://doi.org/10.3912/OJIN. Vol16No01Man01.

Siegrest, J. (1996). Work, self-experience and cardiovascular disease prevention. In K. Orth-Gomer \& N. Schneiderman (Eds.), Behavioural medicine approaches to cardiovascular disease prevention (pp. 87-103). Mahwah, NJ: Erdbaum.
Stamm, B. (2010). The Concise PRoQOL Manual. Pocatello, ID: ProQOL. org. Retrieved from www.proqol.org/uploads/ProQOL_Concise 2ndEd_12-2010.pdf.

Thoma, P., Zalewski, I., von Reventlow, H. G., Norra, C., Juckel, G., \& Daum, I. (2011). Cognitive and affective empathy in depression linked to executive control. Psychiatry Research, 189(3), 373-378. https:// doi.org/10.1016/j.psychres.2011.07.030.

Thomas, J. (2013). Association of personal distress with burnout, compassion fatigue, and compassion satisfaction among clinical social workers. Journal of Social Service Research, 39(3), 365-379. https:// doi.org/10.1080/01488376.2013.771596.

Turgoose, D., \& Maddox, L. (2017). Predictors of compassion fatigue in mental health professionals: A narrative review. Traumatology, 23(2), 172-185. https://doi.org/10.1037/trm0000116.

Valent, P. (1995). Compassion fatigue as STS disorder: Survival strategies: A framework for understanding STS and coping in helpers. In C. Figley (Ed.), Compassion fatigue: Coping with STS disorder in those who treat the traumatized (pp. 21-50). New York, NY: Routledge.

Valent, P. (2002). Diagnosis and treatment of helper stresses, traumas and illness. In C. Figley (Ed.), Treating compassion fatigue (pp. 17-37). New York, NY: Routledge.

Valiente, C., Eisenberg, N., Fabes, R. A., Shepard, S. A., Cumberland, A., \& Losoya, S. H. (2004). Prediction of children's empathy-related responding from their effortful control and parents' expressivity. Developmental Psychology, 40(6), 911-926. https://doi.org/10.1037/ 0012-1649.40.6.911.

Van Dam, N. T., Sheppard, S. C., Forsyth, J. P., \& Earleywine, M. (2011). Self-compassion is a better predictor than mindfulness of symptom severity and quality of life in mixed anxiety and depression. Journal of Anxiety Disorders, 25(1), 123-130. https://doi.org/10.1016/j.janxdis. 2010.08.011.

Wagaman, M. A., Geiger, J. M., Shockley, C., \& Segal, E. A. (2015). The role of empathy in burnout, compassion satisfaction, and secondary traumatic stress among social workers. Social Work (United States), 60(3), 201-209. https://doi.org/10.1093/sw/swv014.

How to cite this article: Coetzee SK, Laschinger HKS. Toward a comprehensive, theoretical model of compassion fatigue: An integrative literature review. Nurs Health Sci. 2018;20:4-15. https://doi.org/10.1111/nhs.12387 